\title{
Detecção ultrassonográfica do ovário fetal em loba-guará (Chrysocyon brachyurus): relato de caso
}

[Ultrasonographic detection of fetal ovary in maned wolf (Chrysocyon brachyurus): case report]

\author{
M.A.R. Feliciano ${ }^{1}$, W.R.R. Vicente $^{2}$, T.F. Motheo $^{1}$, L.C. Padilha ${ }^{3}$, C.G. Candioto ${ }^{3}$ \\ ${ }^{1}$ Aluno de pós-graduação - FCAV-UNESP - Jaboticabal, SP \\ ${ }^{2}$ Faculdade de Ciências Agrárias e Veterinárias - UNESP - Jaboticabal, SP \\ ${ }^{3}$ Médica veterinária residente - FCAV-UNESP - Jaboticabal, SP
}

\begin{abstract}
RESUMO
Identificaram-se, por ultrassonografia, os ovários fetais e o sexo dos fetos em uma loba-guará. Ao exame ultrassonográfico, foi possível identificar estruturas fetais e realizar medidas (relação entre diâmetro cranial e abdominal dos fetos) que indicavam idade fetal de 59 dias. Observaram-se: estruturas torácicas e abdominais formadas, diafragma, membros, estruturas do crânio definidas, coluna vertebral, medula, costelas, batimentos cardíacos normais, movimentação fetal, câmaras e valvas cardíacas, grandes vasos, rins, peristaltismo intestinal e ovários fetais. $\mathrm{O}$ exame ultrassonográfico foi eficaz nessa espécie, pois foi possível observar estruturas fetais para a avaliação da viabilidade fetal e, também, identificou-se a imagem ovariana em um dos fetos, mostrando-se importante para estudos de sexagem fetal.
\end{abstract}

Palavras chave: lobo-guará, gestação, ultrassonografia

\begin{abstract}
The ovaries and the gender of the fetuses in a female maned wolf (Chrysocyon brachyurus) were identified by ultrasound examination. It was possible to identify fetal structures and to determine measures, i.e., cranial and abdominal diameters and these elements indicated 59-day of fetal age. The main visualized structures were diaphragm, members, cranium, spine, marrow, ribs, cardiac chambers and valves, veins and arteries, kidneys, and fetal ovaries. Normal heartbeats and fetal and bowel movements were recorded. The ultrasound examination was an effective method in this species, since enabled the correct observation of the fetal structures as well as viability; in addition, proved to be an important method for fetal sexing determination.
\end{abstract}

Keywords: maned wolf, pregnancy, ultrasonography

\section{INTRODUÇÃO}

O lobo-guará (Chrysocyon brachyurus), membro da família dos canídeos, é conhecido por vários nomes em seu habitat nativo, como boroche, aguará guazu ou lobo de crina. Em vida livre são monoéstricos, pois as fêmeas ciclam uma vez ao ano. O início da estação reprodutiva parece ser responsivo à diminuição do ciclo das luzes, e a maioria dos nascimentos, no hemisfério sul, ocorre de maio a julho. O período de gestação é de 58 a 72 dias, média de 63 - 65 dias, e nascem de dois a cinco filhotes por ninhada (Rodden e
Blakely, 2007). De acordo com Gomes (2007), as ninhadas podem variar de dois a 13 filhotes e a espécie apresenta, normalmente, um ou dois partos por ano.

A detecção da gestação é um dos grandes problemas nos estudos de reprodução e preservação da espécie. As mudanças na aparência das fêmeas durante a gestação são, geralmente, mínimas e, nestes casos, frequentemente exibem discreto aumento abdominal (Rodden e Blakely, 2007). Zambelli e Prati (2006) e Feliciano et al. (2007)

Recebido em 17 de setembro de 2009

Aceito em 9 de fevereiro de 2010

E-mail: marcusfeliciano@yahoo.com.br 
descreveram a importância do exame ultrassonográfico, na rotina veterinária de pequenos animais, quanto ao acompanhamento, ao diagnóstico precoce da gestação, à avaliação da viabilidade fetal e à detecção de possíveis anormalidades da gestação e dos fetos.

Recentemente a ultrassonografia em animais silvestres tornou-se comum na América do Sul. Trata-se de um método seguro, não invasivo e que fornece informações suplementares sobre a anatomia interna e o tamanho de órgãos e estruturas. Uma das mais importantes aplicações é na reprodução, para avaliação do trato genital e, principalmente, para monitoração da gestação (Augusto, 2001). Para avaliação ultrassonográfica, Augusto (2001) recomendou transdutores com frequência que varie de 2 a $10 \mathrm{MHz}$ e também sugerem que a escolha depende da região a ser examinada e do tamanho do animal. Rodden e Blakely (2007) verificaram que o exame ultrassonográfico, nessa espécie, pode ser realizado 30 dias após a cópula, para diagnóstico gestacional.

Em fêmeas gestantes de lobo-guará, os principais indicadores da proximidade do parto são: inquietação dois a três dias antes do parto e agressão ao macho e, muitas vezes, aos tratadores. Mudanças físicas podem incluir aumento abdominal nas duas ou três últimas semanas, desenvolvimento do mamilo, leite nas tetas duas semanas pré-parto e perda de pelos em volta dos mamilos (Rodden e Blakely, 2007).

Este trabalho teve como objetivo principal relatar a detecção, por ultrassonografia, do ovário fetal em uma fêmea de lobo-guará, com a finalidade de identificar o sexo dos fetos, e demonstrar a importância da ultrassonografia como método de diagnóstico gestacional nessa espécie.

\section{CASUÍSTICA}

Uma loba-guará, com $27,25 \mathrm{~kg}$, atropelada em uma rodovia e encaminhada a um serviço de assistência veterinária, foi submetida a exame radiográfico, quando se constatou fratura múltipla e completa no úmero e fratura da pelve, com pronunciação de fragmento ósseo para o interior do canal vaginal. Ainda, por meio desse exame, foi verificada a presença de estruturas no seu abdômen, compatíveis com três imagens fetais.
Após a detecção da gestação, foram realizados: exames físico e obstétrico, dosagem da concentração sérica de progesterona, citologia vaginal com auxílio de suabe estéril e exame ultrassonográfico gestacional, para avaliar a viabilidade da gestação e do feto e estimar a idade dos fetos.

Ao exame físico e obstétrico, o animal apresentou temperatura retal de $37,5^{\circ} \mathrm{C}$, estado geral bom e alerta ao ambiente, e foram verificadas fezes e urina com aspectos e frequência normais durante o período de internação. No dia em que se iniciaram as contrações relacionadas ao trabalho de parto, a temperatura retal era de $39,0^{\circ} \mathrm{C}$. No mesmo dia, foi coletada amostra de sangue para mensuração da concentração sérica de progesterona, e o valor encontrado foi de $3,8 \mathrm{ng} / \mathrm{dL}$ no dia do parto.

Ao exame específico do trato genital, notou-se a presença de edemaciação da região vulvar, com aumento de sensibilidade dolorosa à manipulação. Devido ao histórico de atropelamento e à sensibilidade dolorosa ao exame específico, foi realizada radiografia da região da pelve. Esse exame foi importante para determinar a causa desse processo inflamatório e doloroso, pois foi visibilizada a presença de esquírola óssea na região vulvovaginal.

O material da citologia vaginal foi colhido com o auxílio de um suabe, fixado com metanol e corado em lâmina de vidro por meio de Giemsa. Após a coloração e a fixação do material, foi realizada a microscopia direta em aumentos de 25 e 100 dioptrias, conforme descrito por Feliciano (2006). Ao exame de microscopia direta da citologia vaginal, verificou-se a presença de células do epitélio vaginal queratinizadas e inflamatórias.

Para realização do exame ultrassonográfico, o animal foi previamente tricotomizado no abdômen, entre a região epigástrica e hipogástrica, compreendida entre o apêndice xifoide e os dois últimos pares das glândulas mamárias, e estendendo-se lateralmente na região ventral aos músculos lombares, próximos ao último par de costelas, do lado esquerdo, e sobre os dois últimos pares de costelas, do lado direito. Por meio da contenção dos membros, o animal foi colocado em decúbito lateral, com a cabeça na direção do monitor e o corpo paralelo 
ao aparelho (Feliciano, 2006). Antes do exame, foi utilizado gel específico para a realização de ultrassonografia, Carbogel ULT $^{\odot}$. Foram utilizados transdutores de 5,0 e 7,5MHz.

Ao exame ultrassonográfico, foi possível visibilizar estruturas fetais desenvolvidas, que indicavam idade fetal avançada (terço final da gestação), ao comparar com os dados ultrassonográficos de fetos caninos. Foram visibilizados: área de placentação, estruturas torácicas e abdominais formadas, diafragma, membros, estruturas do crânio definidas, presença de coluna vertebral, medula, costelas, batimentos cardíacos (duas a três vezes o batimento cardíaco materno), movimentação fetal, câmaras e valvas cardíacas, grandes vasos, rins, peristaltismo intestinal e diminuição de líquido intraplacentário (próximo ao parto). No presente relato, foi possível determinar a espessura da placenta e verificar sua característica hemocorial. Essa estrutura apresentava, em média, 1,5cm de espessura (Fig. 1). Um dos principais achados ultrassonográficos foi à visibilização dos ovários fetais e a consequente sexagem fetal.

Nyland e Mattoon (1995) descreveram duas fórmulas para estimar a idade do concepto em cães, por meio do diâmetro parietal (crânio) e abdominal: idade do concepto $=(6 \mathrm{x}$ diâmetro parietal $)+(3 \times$ diâmetro abdominal $)+30$; e idade do concepto $=(7 \times$ diâmetro abdominal $)+$ 29 , com variação de três dias para mais ou para menos, em ambas as fórmulas. Ao usar essas fórmulas e por meio da mensuração dos diâmetros parietal $(2,56 \mathrm{~cm})$ e abdominal $(4,06 \mathrm{~cm})$ dos conceptos, foi possível estimar a fase da gestação média de 59 dias (Fig. 1).
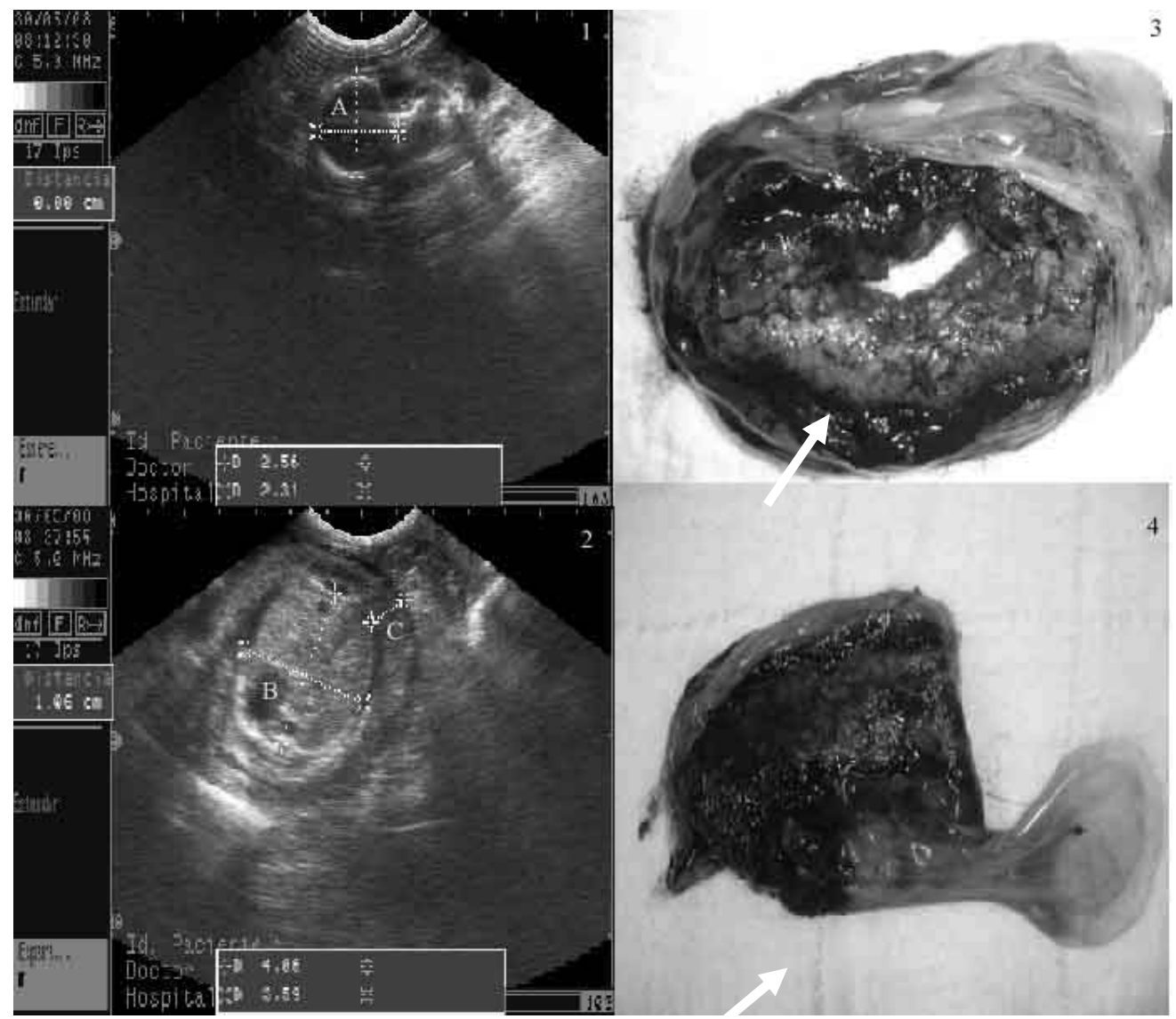

Figura 1. Loba-guará - imagens ultrassonográficas (1) e (2) evidenciando diâmetro parietal fetal (A), diâmetro abdominal fetal (B) e placenta hemocorial (C). Placenta hemocorial (3) e a mesma estrutura seccionada (4) (setas). 


\section{Detecção ultrassonográfica do ovário...}

Por meio da necropsia fetal, foi possível comparar os achados deste procedimento com as imagens ultrassonográficas e avaliar a sensibilidade deste exame nesta espécie. Pode-se visibilizar, nas Fig. 2, 3, 4 e 5, similaridade nas imagens fetais de necropsia e ultrassonográficas.

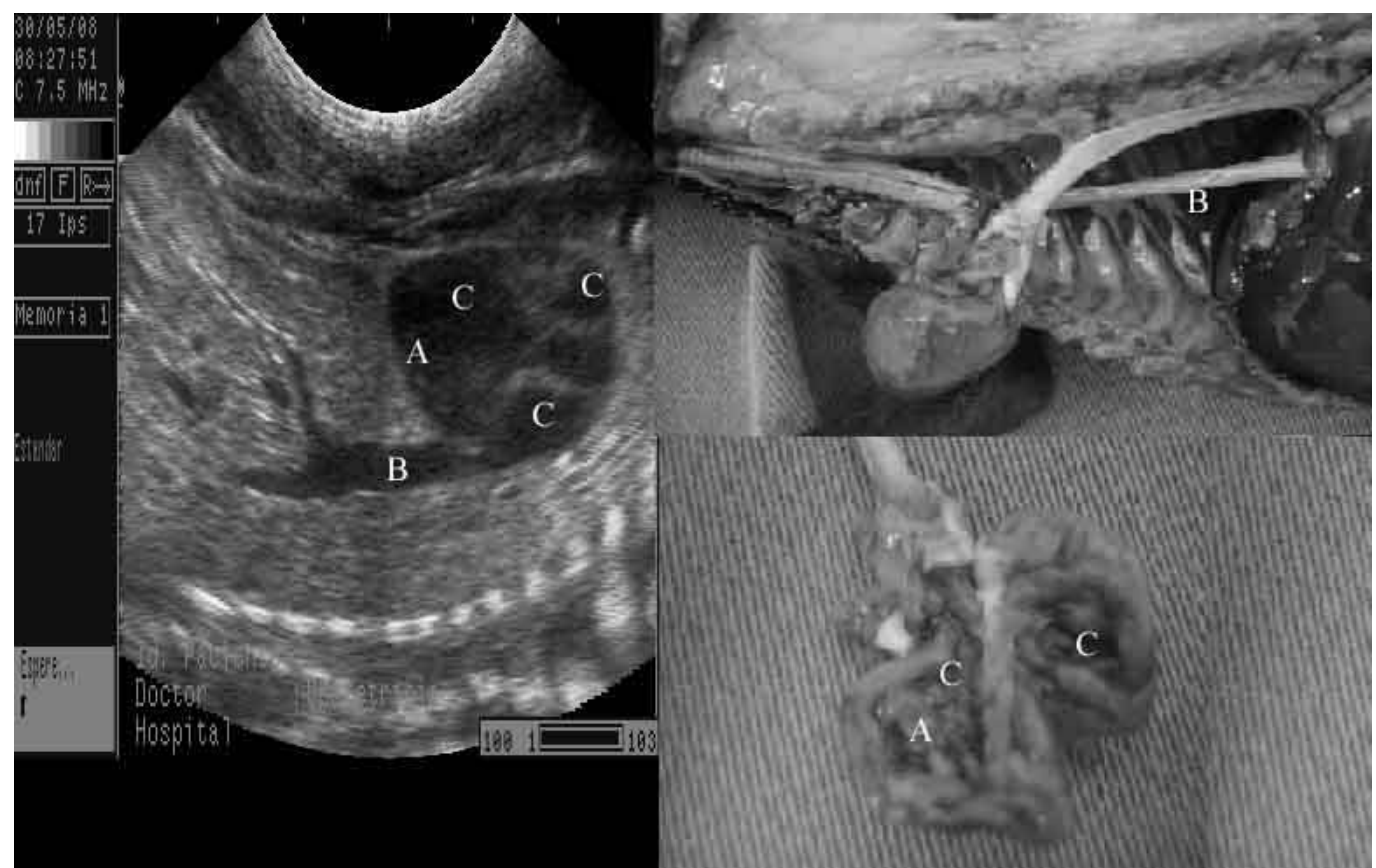

Figura 2. Imagens ultrassonográfica e de necropsia, na avaliação do sistema cardiovascular em feto de loba-guará, evidenciando músculo papilar (A), veia cava caudal (B) e câmaras cardíacas (C).

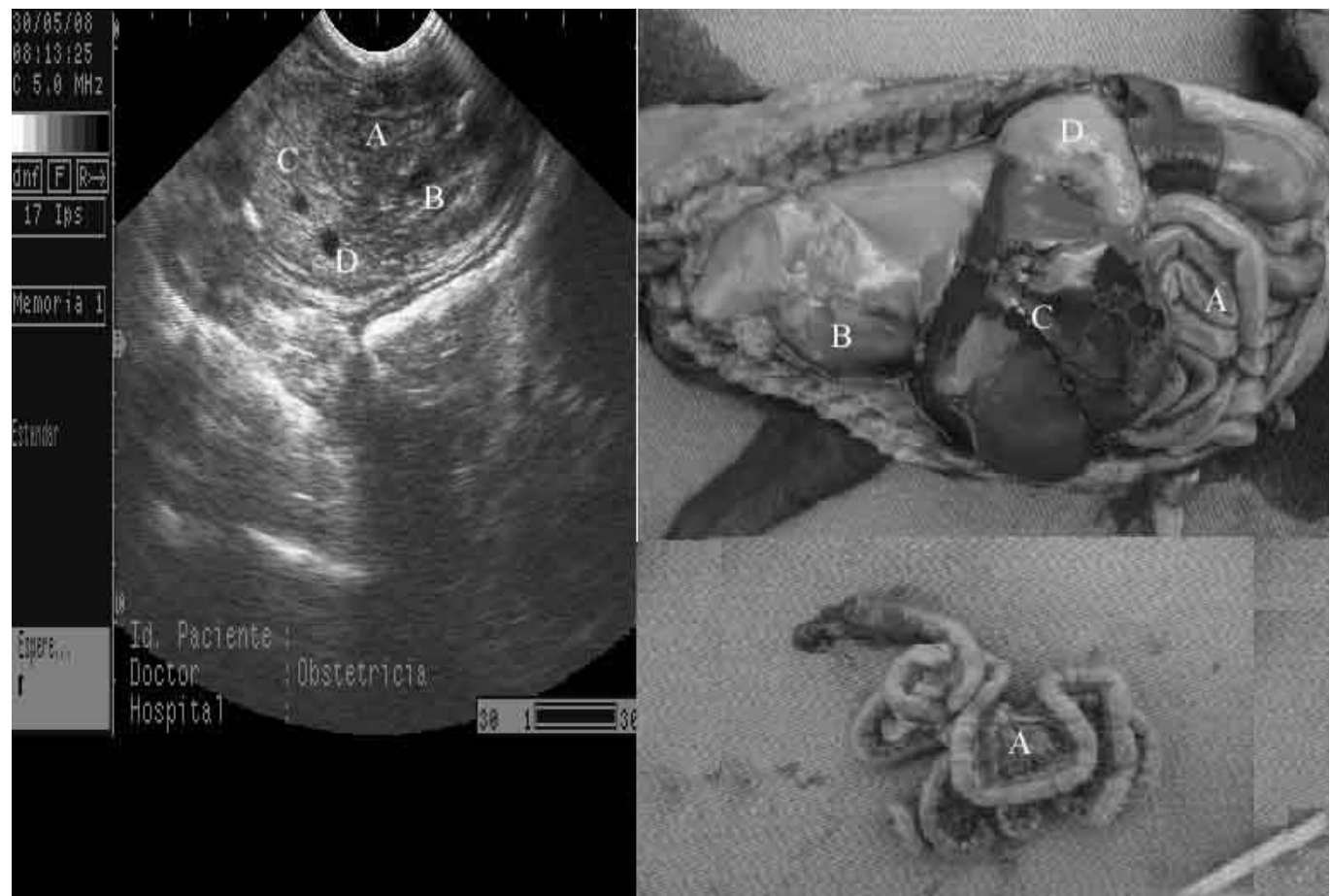

Figura 3. Imagens ultrassonográfica e da necropsia em feto de loba-guará, evidenciando alças intestinais (A), coração (B), fígado (C) e estômago (D). 


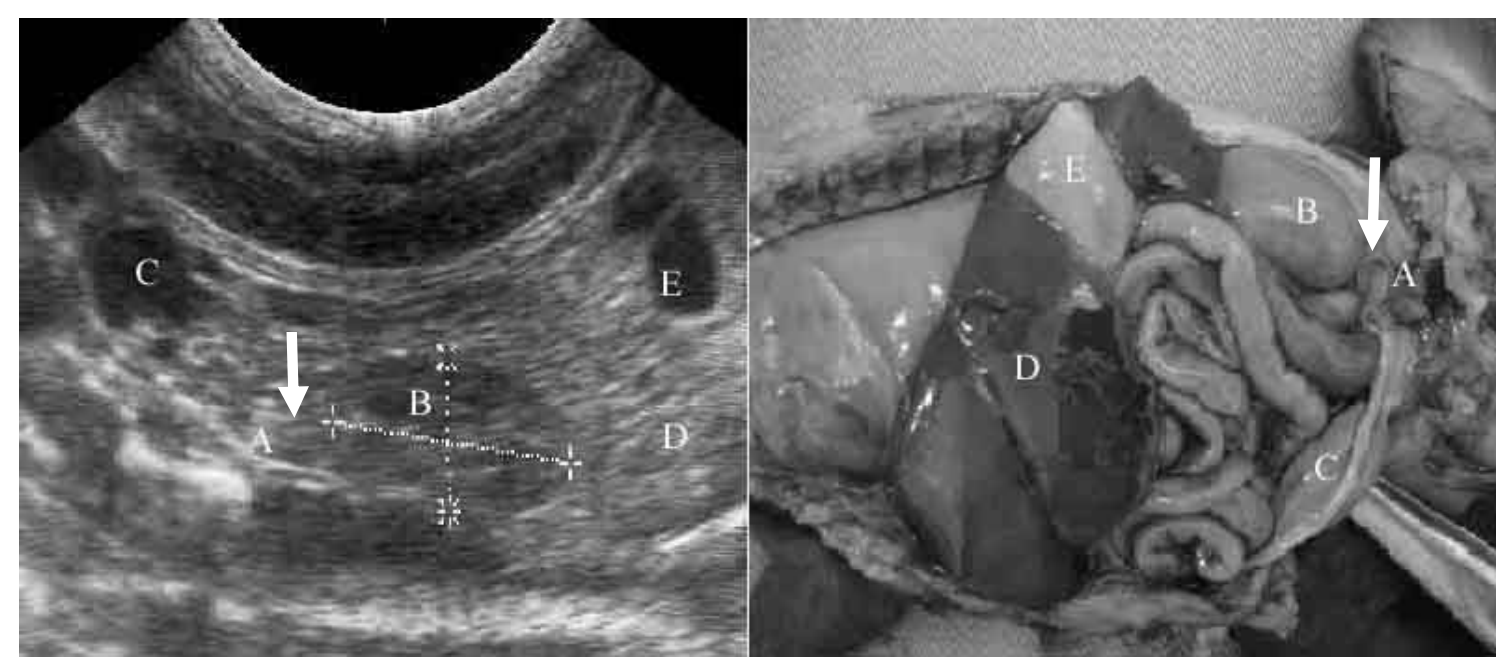

Figura 4. Imagens ultrassonográfica e da necropsia em feto de loba-guará, evidenciando ovário (A/setas), rim (B), bexiga (C), fígado (D) e estômago (E).

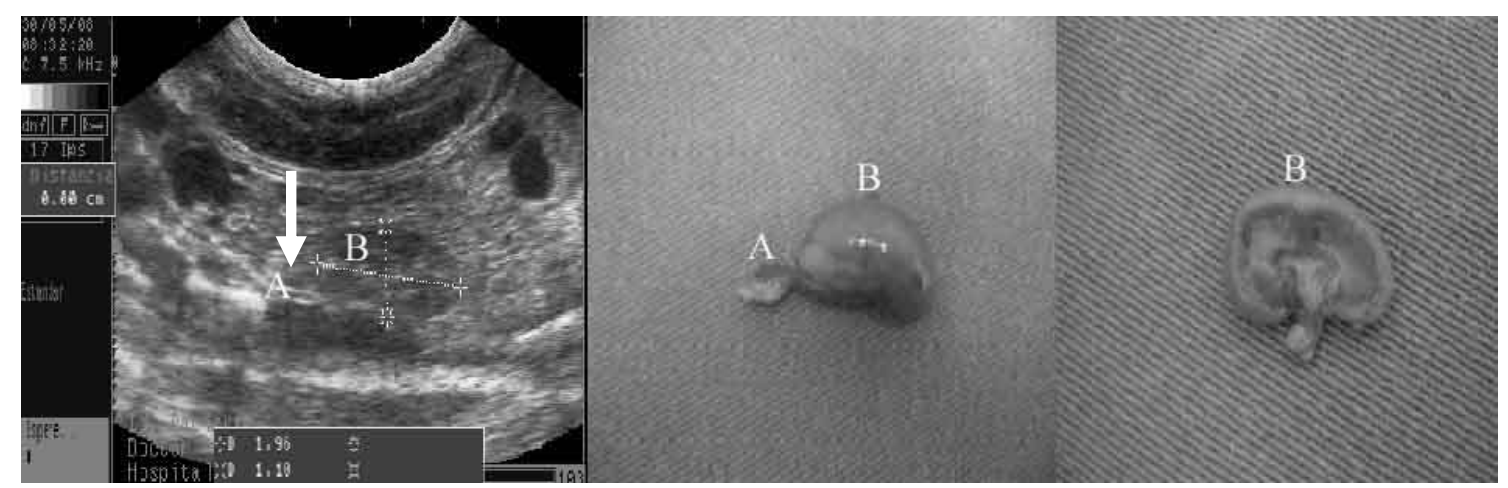

Figura 5. Imagens ultrassonográfica e da necropsia em feto de loba-guará, evidenciando ovário (A/seta) e $\operatorname{rim}(\mathrm{B})$.

O estádio gestacional estimado pela ultrassonografia, mesmo com a presença do desenvolvimento fetal adiantado, indicava que a gestação ainda não estava a termo. A presença de líquido intraplacentário em certa quantidade era o único achado que determinava essa característica gestacional. Entretanto e possivelmente por causa do estresse, o animal iniciou trabalho de parto ao apresentar contrações abdominais, sendo indicada a cesariana devido à presença de esquírola óssea no canal do parto. A fêmea e os filhotes vieram a óbito após o procedimento.

\section{DISCUSSÃO}

No caso clínico presente, a radiografia da região pélvica foi importante para direcionar qual procedimento obstétrico de escolha, pois a presença da esquírola óssea na região vulvovaginal serviu como indicativo para a realização da cesariana. Rodden e Blakely (2007) relataram que as radiografias são sinais importantes de diagnóstico obstétrico, ressaltando que problemas com o uso do ultrassom e do raio-x não têm sido relatados, embora essas técnicas não sejam comumente usadas na rotina. No entanto, deve-se condicionar a fêmea ao uso do ultrassom ou ao exame radiográfico. Dessa forma, esses exames são ferramentas extremamente válidas no manejo reprodutivo da loba-guará.

Rodden e Blakely (2007) descreveram que as dosagens de esteroides e prostágenos podem ser usadas para avaliar a fase reprodutiva da fêmea de lobo-guará, ou seja, por meio da associação dos achados específicos e os valores obtidos pela mensuração da concentração sérica dos hormônios reprodutores, é possível estimar 
períodos do ciclo estral e da gestação. Soto et al. (2004) verificaram secreção pulsátil de hormônio luteinizante, estrógeno e progesterona durante o ciclo estral dessa espécie. Em cadelas, valores de progesterona acima de $2 \mathrm{ng} / \mathrm{dL}$ podem indicar ovulação, com a progesterona atingindo níveis acima de 32ng/dL no diestro (Maia et al., 2008). Esses valores de referência podem ser extrapolados para as fêmeas de lobo-guará.

Maia et al. (2008) demonstraram que os níveis de progesterona de lobas-guará variam com a estação do ano. Esses autores verificaram valores de progesterona de $17,1 \pm 6,0 \mathrm{ng} / \mathrm{dL}$, no outono, e de 1,7 $\pm 0,3 \mathrm{ng} / \mathrm{dL}$, no inverno, estando associados com o período fértil dos animais. Ao se comparar o valor obtido da concentração de progesterona nesta fêmea, 3,8ng/dL, com valores em cadelas, pode-se concluir que o animal estava em fase final de gestação.

Segundo Velloso et al. (1998) e Gobello e Corrada (2003), a duração e as características das fases do ciclo estral, a pseudogestação e a morfologia do oócito na loba-guará são semelhantes às da cadela e às de outras canídeas. Noakes (1996) citou que, no período de estro, à microscopia direta, verifica-se a presença de células inflamatórias, pequena quantidade de células epiteliais nucleadas e de hemácias. Esses achados microscópicos na loba-guará são relacionados, de acordo com Noakes (1996), com a fase do estro, em cadelas, em que o valor da concentração sanguínea de progesterona está diminuído, semelhante ao período próximo ao parto. No entanto, vale considerar que, no presente trabalho, observou-se esquírola óssea na região vulvovaginal, com reação inflamatória que altera a celularidade típica do diestro.

Os resultados ultrassonográficos obtidos confirmam os já citados por Kustritz (2005) e Feliciano et al. (2007), que comentaram a visibilização, ao exame ultrassonográfico após o $59^{\circ}$ dia da gestação, da área de placentação, das estruturas torácicas e abdominais formadas, do diafragma, dos membros, das estruturas definidas do crânio, da presença de coluna vertebral, da medula, das costelas, dos batimentos cardíacos (duas a três vezes o batimento cardíaco materno), da movimentação fetal, das câmaras e valvas cardíacas, dos grandes vasos, dos rins, do peristaltismo intestinal e da diminuição de líquido intraplacentário (próximo ao parto). A idade fetal estimada de 59 dias de gestação condiz com o cálculo realizado e os achados ultrassonográficos do desenvolvimento fetal em cães com a idade fetal determinada (Feliciano et al., 2007).

A visibilização ultrassonográfica do ovário fetal em loba-guará é inédita na literatura veterinária e pode ser utilizada como método de determinação do sexo dos fetos pelos ultrassonografistas veterinários. A literatura cita apenas que a determinação do sexo fetal, ao exame ultrassonográfico, pode ocorrer pela primeira vez dos 38 aos 43 dias de gestação, em gatas (Zambelli e Prati, 2006). Esses autores identificaram a genitália externa, o prepúcio, o escroto e a vulva, nos fetos. Essa identificação pode ser dificultada pelos fluidos fetais e pela cauda. Não há relatos de imagem das gônadas fetais para determinação do sexo, reafirmandose, dessa forma, a relevância desse achado no presente estudo.

\section{CONCLUSÕES}

A detecção ultrassonográfica de ovários fetais em loba-guará, possivelmente, constitui um dado relevante para estudos de sexagem fetal. Essa técnica é indicada na avaliação gestacional em animais dessa espécie, pois os achados de necropsia dos fetos foram coincidentes com as imagens ultrassonográficas obtidas. Dessa forma, considera-se a realização desse exame como um método de diagnóstico auxiliar no acompanhamento gestacional em lobas-guará e sua importância para preservação da espécie.

\section{REFERÊNCIAS BIBLIOGRÁFICAS}

AUGUSTO, A.Q. Ultrasonography in South American wild animals. In: FOWLER, M.E.; CUBAS, Z.S. Biology, medicine and surgery of South American wild animals. Iowa: Iowa State University Press, 2001. 536p.

FELICIANO, M.A.R.; MUZZI, L.A.L.; LEITE, C.A.L. et al. Ultrassonografia bidimensional convencional, de alta resolução e tridimensional no acompanhamento da gestação em cadela. Arq. Bras. Med. Vet. Zootec., v.59, p.1333-1337, 2007. 
FELICIANO, M.A.R. Ultrassonografia bidimensional convencional, de alta resolução $e$ tridimensional no acompanhamento da gestação em cadela. Lavras: UFLA, 2006. 41p.

GOBELLO, C.; CORRADA, Y. Biotechnology in canine reproduction: an update. Analecta Vet., v. 23, p.30-37, 2003.

GOMES, M.S. Carnivora, Canidae (lobo-guará, cachorro-do-mato, raposa-do-campo). In: CUBAS, Z.S.; SILVA, J.C.R.; CATÃO-DIAS, J.L. Tratado de animais selvagens: medicina veterinária. São Paulo: Roca, 2007. p.492-504.

KUSTRITZ, M.V.R. Pregnancy diagnosis and abnormalities of pregnancy in the dog. Theriogenology, v.64, p.755-65, 2005.

MAIA, O.B.; JÁCOMO, A.T.A.; BRINGEL, B.A. et al. Comparison of serum hormone levels of captive and free-living maned wolves Chrysocyon brachyurus. Braz. J. Med. Biol. Res., v.41, p.176-179, 2008.

NOAKES, D.E. Pregnancy and its diagnosis. In: ARTUHR, G.H.; NOAKES, D.E.; PEARSON, $\mathrm{H}$. et al. Veterinary reproduction and obstetrics. 7.ed. London: W.B. Saunders, 1996. p.63-109.
NYLAND, G.T.; MATTOON, J.S. Ultrasonography of the genital system. In: Veterinary diagnostic ultrasound. Philadelphia: W.B. Saunders, 1995. p.141-164.

RODDEN, M.; BLAKELY, M. Manual de manejo de lobo-guará. São Paulo: MWSSP, 2007. 99p.

SOTO, M.A.; MENDEZ, A.S.; PULIDO, J.R. et al. Valoración de hormonas esteróides em heces de uma pareja de lobo mexicano (Canis lúpus baileyi) em cautiverio. Acta Zool. Mex., v.20, p.187-196, 2004.

VELLOSO, A.L.; WASSER, S.K.; MONFORT, S.L. et al. Longitudinal fecal steroid excretion in maned wolves (Chrysocyon brachyurus). Gen. Comp. Endocrinol., v.112, p.96-107, 1998.

ZAMBELLI, D.; PRATI, F. Ultrasonography for pregnancy diagnosis and evaluation in queens. Theriogenology, v.66, p.135-144, 2006. 